\title{
Severe Primary Cytomegalovirus Pneumonia in a 5-year-old Immunocompetent Child
}

Sir,

Life threatening cytomegalovirus (CMV) infection mostly occurs in the fetus, newborn, infant or immunodeficient individuals. In the immunocompetent, it is usually silent or may present as mononucleosis like syndrome. ${ }^{1}$ Here we describe an immunocompetent and previously healthy child who suffered from severe primary CMV pneumonia. A 5-year-old boy presented with fever, cough, dyspnea, hypoxemia and mild elevations of glutamic oxalacetic transaminaseÿ lactate dehydrogenase and creatinine kinase on March 26, 2009. Chest radiograph showed multiple parenchymal consolidations. The diagnosis of primary CMV pneumonia was confirmed by positive PCR $\left(2.4 \times 10^{4}\right)$ from bronchoalveolar lavage at admission, with seroconversions of CMV IgM and IgG after one month of hospitalization. Bacterial cultures of blood, endotracheal secretion, pleural fluid and urine were negative at admission. Endotracheal secretion PCR and/or serology IgM tests for influenza, parainfluenza, adenovirus, respiratory syncytial virus, metapneumovirus, herpes virus, enterovirus general type, enterovirus 71, coxsackie virus A16, HIV, mycoplasmal pneumonia and chlamydia pneumoniae were all negative. His CBC had shown WBC count of $6.9 \times 10^{9} / \mathrm{L}$ with $3.6 \times 10^{9} / \mathrm{L}$ of neutrophils, $2.8 \times 10^{9} / \mathrm{L}$ of lymphocytes, $123 \mathrm{~g} / \mathrm{L}$ of $\mathrm{Hb}$ and $156 \times 10^{9} / \mathrm{L}$ of platelets at onset. During the hospitalization, his lymphocytes decreased to $(0.67 \pm 0.26)$ $\times 10^{9} / \mathrm{L}$. The T cell subset analysis showed low ratio of CD4/ CD8 $\left(\mathrm{CD}^{+}\right.$65.1\%, $\mathrm{CD}^{+} 28.0 \%, \mathrm{CD}^{+} 32.6 \%, \mathrm{CD}^{+}{ }^{+} \mathrm{CD}^{+}$/ $\mathrm{CD}^{+}{ }^{+} \mathrm{CD}^{+}$: $\left.0.86: 1\right)$. Humoral immune function analysis was normal $(\operatorname{IgG} 6.11 \mathrm{~g} / \mathrm{L}, \mathrm{IgA} 0.62 \mathrm{~g} / \mathrm{L}$, IgM 0.76g/L, IgE 12.9g/L, complement C3 0.52g/L, complement C4 0.16g/L). Chest radiograph disclosed "white lung" during the second week. He had received antibiotics including cefoperazone/ sulbactam, panipenem, meropenem, linezolid, amphotericin B liposome and voriconazole. He rapidly developed pulmonary failure and was supported with ventilator, extracorporeal membrane oxygenator and hemoperfusion. He developed ventilator associated pneumonia, and died of burkhoderia sepsis after 35 days of hospitalization. Autopsy was not done.

We agree with Cunha $\mathrm{BA}^{2}$ that lung infection accompanied by hepatic involvement is an important clue to the diagnosis. The present case is also unique for the following two reasons not previously described in immunocompetent host. Firstly, CMV PCR is more reliable than the CMV-IgM at the onset of CMV infection. CMV IgM can be demonstrated transiently (4 16 weeks) during the acute symptomatic phase in immunocompetent hosts. CMV-IgM may be negative at onset of CMV infection. Eddleston $\mathrm{M}$ reported, that out of 34 cases of severe CMV infection in immunocopetent host, only 23 patients were confirmed by CMV-IgM. ${ }^{3}$

Secondly, CMV caused lymphopenia in this immunocompetent host. He was a previously healthy boy with normal complete blood count at onset, but developed lymphopenia, which suggested that the lymphopenia was caused by severe CMV infection. CMV can infect both hematopoietic progenitor cells and stromal elements; lymphopenia can be due to both direct inhibition of progenitor cell growth and the failure of stem cell self-renewal. ${ }^{4}$ Thus, CMV PCR is more reliable to make diagnosis than IgM at onset and it can cause lymphopenia; when viral pneumonia is accompanied by increased serum transaminases, and common respiratory pathogens are ruled out, CMV should be considered.

Yinghu Chen, Yongmin Tang, Chenmei Zhang, Ru Lin, Tao Liu and Shiqiang Shang Intensive Care Unit, Zhejiang Key Laboratory for Neonatal Diseases, Children's Hospital of Zhejiang University Medical College, Hangzhou 310003, China. E-mail: shangsq33@sian.com [DOI-10.1007/s12098-010-0086-1]

\section{REFERENCES}

1. Rafailidis PI, Mourtzoukou EG, Varbobitis IC, Falagas ME. Severe cytomegalovirus infection in apparently immunocompetent patients: a systematic review. Virol J 2008; 5: 47.

2. Cunha BA, Pherez F, Walls N. Severe cytomegalovirus (CMV) community-acquired pneumonia (CAP) in a nonimmunocompromised host. Heart Lung 2009; 38: 243-248.

3. Eddleston M, Peacock S, Juniper M, Warrell DA. Severe cytomegalovirus infection in immunocompetent patients. Clin Infect Dis 1997; 24: 52-56.

4. Sing GK, Ruscetti FW. The role of human cytomegalovirus in haematological diseases. Baillieres Clin Haematol 1995; 8: 149163. 